\title{
Supramolecular Analysis of a Multi-Cation Hydrated Decavandate Salt
}

\author{
Joseph P. Haller ${ }^{1}$, Kenneth J. Haller ${ }^{2}$, and Aungkana Chatkon ${ }^{3}$ \\ ${ }^{1}$ Home School, P.O. Box 43, Chom Surang, Nakhon Ratchasima 30001 Thailand \\ ${ }^{2}$ School of Chemistry, Suranaree University of Technology, Nakhon Ratchasima 30000 Thailand, \\ ken.haller@gmail.com \\ ${ }^{3}$ Chemistry Program, Faculty of Science and Technology, Nakhon Ratchasima Rajabhat University, \\ Nakhon Ratchasima 30000 Thailand, tuy448@yahoo.com
}

As part of our studies incorporating metformin with other compounds used for diabetes therapy ${ }^{[1,2]}$, a salt of decavanadate, $\mathrm{V}_{10} \mathrm{O}_{28}{ }^{6-}$, containing mixed organic cations has been synthesized by heating metformin, picolinic acid, and sodium metavanadate in aqueous solution at $\mathrm{pH} 4$ and $60{ }^{\circ} \mathrm{C}$ (similar to previous method ${ }^{[2]}$ ), followed by keeping the solution at RT overnight to give an orange crystalline product. The product was characterized by single crystal X-ray diffraction, vibrational spectroscopy, and thermal analysis. As generally observed, the $\mathrm{V}_{10} \mathrm{O}_{28}{ }^{6-}$ anion lies on an inversion center ${ }^{[1,2]}$. Guanylurea cation, $\mathrm{HGU}^{+}$, forms from metformin in the synthesis under similar conditions to those in the previous report ${ }^{[2]}$. The charge of the six-minus decavanadate anion is balanced by two diprotonated metformin cations, $\mathrm{H}_{2} \mathrm{Met}^{2+}$, one disordered $\mathrm{HGU}^{+}$cation, and one hydronium ion for which the proton could not be found. There are eight waters of hydration per $\mathrm{V}_{10} \mathrm{O}_{28}{ }^{6-}$. The salt exhibits a complex three-dimensional hydrogen bonding network involving water molecules, cationanion, and cation-cation interactions through $\mathrm{O}_{\mathrm{w}}-\mathrm{H} \cdots \mathrm{O}, \mathrm{N}-\mathrm{H} \cdots \mathrm{O}$, and $\mathrm{C}-\mathrm{H} \cdots \mathrm{O}$ intra- and inter-molecular interactions.

The metformin occurs in the present compound in the diprotonated form, $\mathrm{H}_{2} \mathrm{Met}^{2+}$, even though the dominant solution species at the synthesis $\mathrm{pH}$ is the monoprotonated, $\mathrm{HMet}^{+}$, similar to other recently reported compounds ${ }^{[3]}$. The TGA result shows loss of $\mathrm{HGU}^{+}$at lower temperature along with the waters of hydration, perhaps understandable because the $\mathrm{HGU}^{+}$ exhibits disorder with four overlapping positions resulting in four different supramolecular environments within the same space. The disorder and the supramolecular environment of the multiple cations, the decavandate anions, and the waters of hydration will be discussed. Crystal data: monoclinic, $P 2_{1} / c, Z^{\prime}=2$, orange plate crystals; $\mathrm{C}_{10} \mathrm{H}_{49} \mathrm{~N}_{14} \mathrm{O}_{38} \mathrm{~V}_{10} ; T=296.2 \mathrm{~K}$; $\theta_{\max }=26.41^{\circ} ; R_{1}=0.035, w R_{2}=0.084 ; \rho_{\max }=0.76 \mathrm{e} \AA^{-3}$.

\section{References:}

1. Chatkon; Chatterjee; Sedgwick; Haller; Crans. Eur. J. Inorg. Chem. 2013 (10-11) 1859-1868.

2. Chatkon; Barres; Samart; Boyle; Haller; Crans. Inorg. Chim. Acta 2014 420, 85-91.

3. Sanchez-Lombardo; Sanchez-Lara, et al. Eur. J. Inorg. Chem. 2014 4581-4588. 